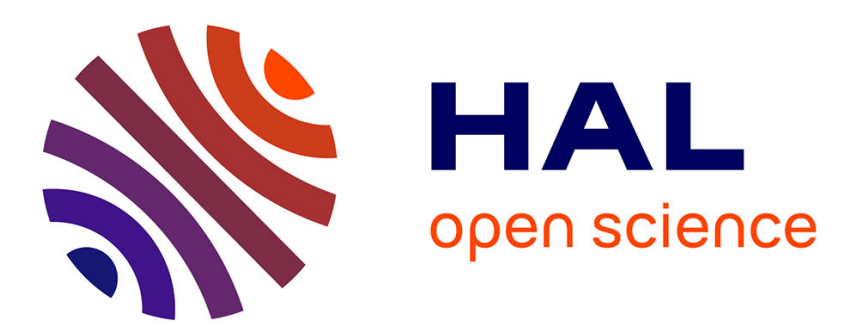

\title{
Une base cartographique de données phytoécologiques issue des relevés de catalogues de types de stations forestières.
}

\author{
Michel Bartoli, Gérard Largier, S. Abgrall, E. Luxcey
}

\section{To cite this version:}

Michel Bartoli, Gérard Largier, S. Abgrall, E. Luxcey. Une base cartographique de données phytoécologiques issue des relevés de catalogues de types de stations forestières.. Revue forestière française, 1999, 51 (1), pp.47-56. 10.4267/2042/5416 . hal-03443428

HAL Id: hal-03443428

https://hal.science/hal-03443428

Submitted on 23 Nov 2021

HAL is a multi-disciplinary open access archive for the deposit and dissemination of scientific research documents, whether they are published or not. The documents may come from teaching and research institutions in France or abroad, or from public or private research centers.
L'archive ouverte pluridisciplinaire HAL, est destinée au dépôt et à la diffusion de documents scientifiques de niveau recherche, publiés ou non, émanant des établissements d'enseignement et de recherche français ou étrangers, des laboratoires publics ou privés. 


\title{
technique et forêt
}

\section{UNE BASE CARTOGRAPHIQUE DE DONNÉES PHYTO-ÉCOLOGIQUES ISSUE DES RELEVÉS DE CATALOGUES DE TYPES DE STATIONS FORESTIĖRES}

\author{
M. BARTOLI - G. LARGIER - S. ABGRALL - E. LUXCEY
}

Pour réaliser un catalogue des types de stations forestières suivant la méthode largement usitée en France (Brêthes, 1989), des relevés phyto-écologiques nombreux, précis et fiables sont réalisés suivant un échantillonnage stratifié permettant d'appréhender la variabilité écologique régionale.

Les très nombreuses données qui en résultent servent à établir une synthèse (le catalogue), mais les informations brutes initiales sont ensuite perdues de vue. En les réutilisant, nous disposons, pour chaque station élémentaire (une à plusieurs centaines par catalogue), des coordonnées géographiques de chaque point de relevé auquel il sera possible d'associer toutes les informations écologiques notées sur le terrain.

En associant ces données botaniques et écologiques à un logiciel de cartographie, l'informatique, outil principal de ce travail, va permettre de récupérer une connaissance écologique originale pour en faciliter l'accès et une utilisation simple et conviviale.

Sans entrer dans le détail des travaux effectués (1), nous présentons la démarche réalisée et le contenu de la base cartographique de données phyto-écologiques mise en place. Elle concerne une partie des Pyrénées couvrant environ 160000 ha de forêts. 


\section{LES OBJECTIFS}

Cette base de données veut être :

\section{- Un document de connaissance}

Elle est un vrai point de repère. Les forestiers de terrain vont pouvoir visualiser les stations, repérer sols et espèces. Les scientifiques y trouveront ces données localisées sur des territoires en fait peu connus notamment sur le plan de la phytosociologie comme l'a montré Savoie (1996).

\section{- Un document d'alerte et un outil d'évaluation patrimoniale}

Elle attire l'attention sur des espaces, habitats et espèces sensibles et peut permettre d'orienter des recherches en ces domaines.

Pour d'autres utilisateurs, ce type de document, bien que partiel, doit pouvoir être un bon outil : un conservatoire botanique peut y trouver une riche matière jusqu'alors inaccessible, les responsables de la carte pédologique de la France peuvent voir là, hors espaces cultivés, un document inespéré.

Alors que nous étions en train de la créer, cette base de données a déjà servi deux fois :

- pour renseigner le Laboratoire de Génétique forestière de I'ONF qui cherchait Sorbus torminalis et ses hybrides. L'un de ceux-ci, non signalé dans la région, avait été trouvé dans un relevé de l'Ariège.

- un chercheur en entomologie (de l'INRA à Rennes) cherchait Prunus padus, hôte alterne d'un puceron parasite des céréales. Il a eu une réponse utilisable immédiatement pour réaliser une partie d'un échantillonnage.

\section{LES CATALOGUES UTILISÉS}

Nous avons utilisé les relevés de catalogues traitant des forêts des Pyrénées centro-occidentales et de leurs piémonts. Globalement, les relevés correspondent à un point tous les 120 ha de forêts environ. II est important de souligner que ces relevés se présentaient déjà sur format informatique qu'il a suffi d'adapter à la structure de notre base de données.

\begin{tabular}{|c|l|c|}
\hline \multicolumn{1}{|c|}{ Auteur (1) } & \multicolumn{1}{c|}{ Zone } & \multicolumn{1}{c|}{$\begin{array}{c}\text { Nombre } \\
\text { de relevés }\end{array}$} \\
\hline Sylvie Baffalio . . . . . & Massif des Arbailles (Pyrénées-Atlantiques) & 130 \\
\hline Laurent Larrieu . . . . . & Coteaux entre Oloron et Nay (Pyrénées-Atlantiques) & 83 \\
\hline Daniel Pons . . . . . . . & Massif de Pointis-Inard (Haute-Garonne) & 106 \\
\hline Jean-Marie Savoie . . . E & $\begin{array}{l}\text { Étage collinéen et étage montagnard des "Pyrénées centrales” } \\
\text { (Ariège, Haute-Garonne, Hautes-Pyrénées) (2) }\end{array}$ & 1005 \\
\hline Bernard Vignes . . . . & Petites Pyrénées (Ariège) & 84 \\
\hline & \multicolumn{1}{c}{ Total } & 1408 \\
\hline
\end{tabular}

(1) Voir la bibliographie (p. 55).

(2) Ce catalogue intègre les 81 relevés de celui de Largier (1988) en forêt domaniale de Cagire (Haute-Garonne). 


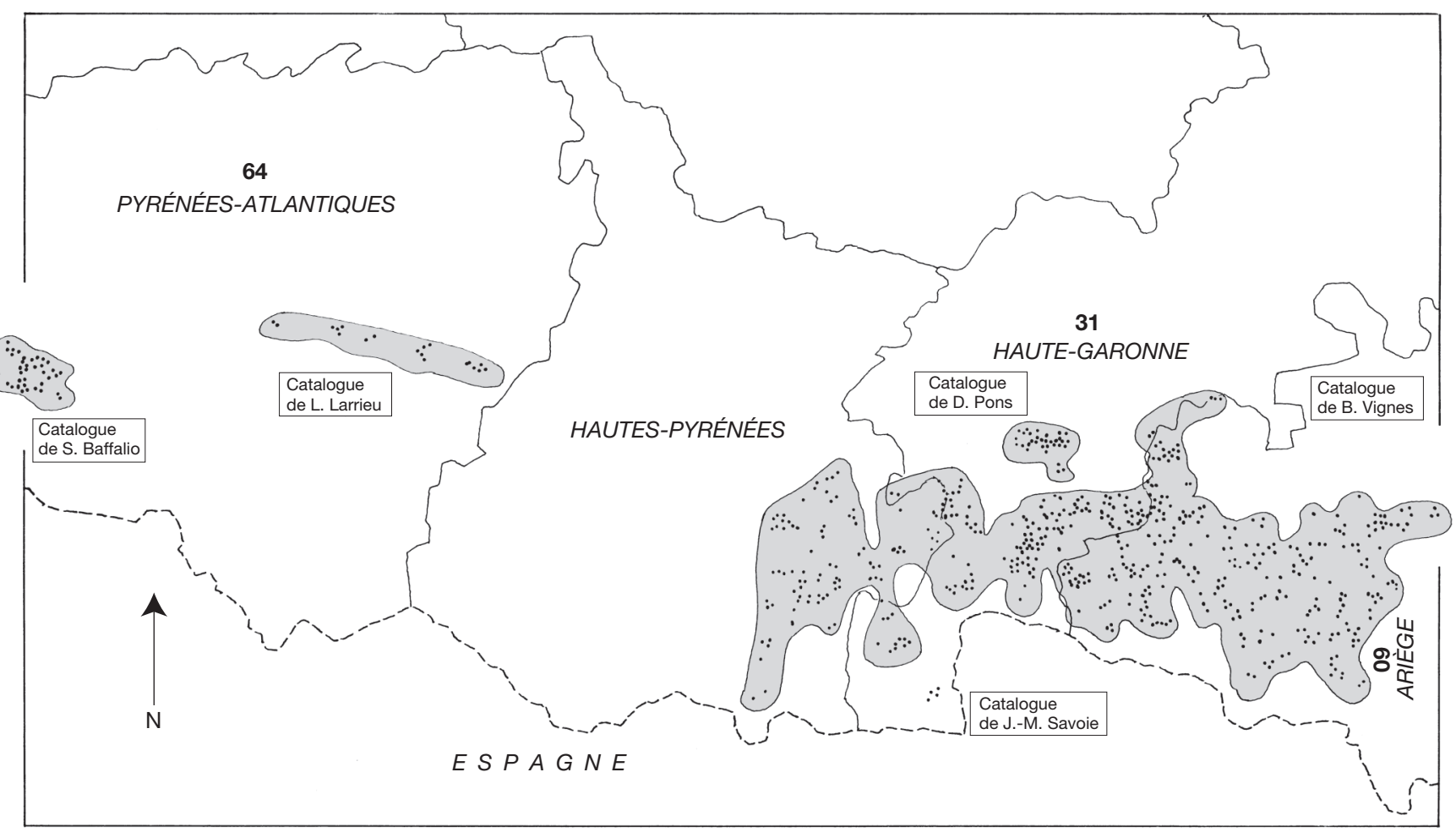

Figure 1

CROQUIS DE SITUATION DES ZONES ÉTUdiÉES PAR LES CATALOGUES

Au total, la base ainsi constituée contient environ 170000 données. Ces informations en quantité relativement considérable dans des domaines où elles sont rares et longues à recueillir sont ainsi remises à disposition de tous sous une forme facilement accessible. Actuellement, deux autres catalogues sont en cours de prise en compte : une étude un peu ancienne de Tierle (1978) sur les sapinières des Hautes-Pyrénées et un travail récent de Goyheneix (1995) sur la forêt communale d'Oloron-Sainte-Marie.

II convient de noter l'intérêt d'intégrer des relevés anciens qui permettent un retour sur le terrain, aux mêmes endroits, 20 ou 30 ans après. Cela pourra aider à mettre en évidence des effets de modifications, climatiques ou autres, comme ont pu le faire Dupouey et al. (1998) dans les Alpes.

\section{LES INFORMATIONS DE LA BASE DE DONNÉES}

La base de données permet d'éditer :

- des cartes sur transparents (en coupures de format A4, à l'échelle souhaitée, au $1 / 25000$ en standard), comportant les points de relevés et des points de repères. Sur ces cartes, sont indiqués, uniquement, les points des relevés résultant de la commande passée par un utilisateur (par exemple : "les relevés situés en Ariège et comprenant Paris quadrifolia"). 
- les fiches associées aux points de la carte. Cette fiche restitue la totalité de l'information notée pour établir le catalogue et des descripteurs ajoutés ultérieurement, comme, bien entendu, le type de station.

Le transparent fourni est, tout simplement, posé sur le document de travail : aménagement forestier, zone d'étude d'impact de route...

\section{Les indications de la fiche de relevé}

La fiche associée à chaque point se présente sous la forme suivante (modifiée pour servir d'exemple) :

Figure 2

EXEMPLE D'UNE FICHE ASSOCIÉE À UN POINT DE RELEVÉ

Catalogue de référence

\section{Point $\mathrm{n}^{\circ} \mathbf{1 8}$}

\begin{tabular}{|l|c|}
\hline Observateur $\ldots \ldots \ldots$ & J.-M. Savoie \\
\hline Date du relevé $\ldots \ldots \ldots$ & $11 / 06 / 91$ \\
\hline
\end{tabular}

\begin{tabular}{|l|c|}
\hline \multicolumn{2}{|c|}{ Références géographiques } \\
\hline Carte IGN $\ldots \ldots \ldots \ldots$ & 20.47 O \\
Région IFN $\ldots \ldots \ldots$ & front pyrénéen \\
Exposition $\ldots \ldots \ldots \ldots$ & NNE \\
Altitude $(\mathrm{m}) \ldots \ldots \ldots$ & 930 \\
Étage bioclimatique $\ldots$ & montagnard inférieur \\
Topographie ....... & mi-pente \\
Coord. Lambert III $(\mathrm{m}) \mathrm{X}$ & 444074 \\
Coord. Lambert III $(\mathrm{m}) \mathrm{Y}$ & 3069169 \\
\hline
\end{tabular}

\begin{tabular}{|l|c|}
\hline \multicolumn{2}{|c|}{ Références administratives } \\
\hline $\begin{array}{l}\text { Département } \ldots \ldots \ldots \ldots \\
\text { Commune de situation . }\end{array}$ & 09 \\
\hline
\end{tabular}

\begin{tabular}{|c|c|}
\hline \multicolumn{2}{|c|}{ Géologie/Pédologie } \\
\hline Substratum . . . . . & schiste ardoisier \\
\hline Matériau parental ... & altérite limoneuse \\
\hline Forme d'humus .... & mésomull \\
\hline Type de sol .... & $\begin{array}{l}\text { brunisol ou néoluvisol } \\
\text { mésosaturé }\end{array}$ \\
\hline Épaisseur du sol . . . & 80 à $120 \mathrm{~cm}$ \\
\hline
\end{tabular}

Type de station ....... 43M

Habitat . . . . . . . . . . 41.141
Pyrénées centrales

\begin{tabular}{|c|c|c|c|c|c|}
\hline \multicolumn{6}{|c|}{ Relevé botanique } \\
\hline Strate & $\begin{array}{c}\text { Espèce } \\
\text { (par strate et ordre } \\
\text { alphabétique) }\end{array}$ & $A / D$ & $\mathrm{GEI}^{*}$ & $\mathrm{GE}^{\star \star}$ & Alerte $^{\star \star \star}$ \\
\hline A & Fagus sylvatica . . . . & 4 & $\mathrm{tla}$ & tal lhs & \\
\hline$A$ & Fraxinus excelsior . . & 1 & $\mathrm{nN}$ & $\mathrm{n}$ hs & \\
\hline A & Malus sylvestris ... & + & $\mathrm{n}$ la & tla & $\bullet$ \\
\hline$A$ & Populus tremula ... & 1 & a m & nla m & \\
\hline A & Prunus avium ..... & 1 & $\mathrm{n}$ am & nla $m$ & \\
\hline a & Cornus sanguinea . . & 1 & $\mathrm{~b}$ & $\mathrm{ccl} \mathrm{m}$ & \\
\hline a & Corylus avellana ... & 2 & $\mathrm{n}$ la & n lhs & \\
\hline $\mathrm{h}$ & Athyrium filix-femina & 1 & $\mathrm{ah}$ & $\mathrm{n}$ hs & \\
\hline $\mathrm{h}$ & Blechnum spicant . . & 1 & A la & nla m & \\
\hline $\mathrm{h}$ & Carex sylvatica . . . & + & $\mathrm{n}$ am & nma hs & \\
\hline
\end{tabular}

* Groupe d'espèces indicatrices de la Flore forestière française

** Groupe écologique du catalogue de référence

*** Information sur la valeur patrimoniale 


\section{La notice de la base de données}

La base de données est accompagnée d'une notice d'une trentaine de pages. Cette notice comprend :

- une partie générale qui définit les termes, codes ou sigles utilisés, rappelle quelques définitions.

- un bref dossier propre à chaque catalogue. Ce dossier n'est là que pour fournir la signification des termes spécifiques au catalogue et qui sont contenus dans les fiches. C'est le cas des codifications des groupes écologiques et des types de stations, des correspondances entre stations et habitats.

Nous avons été amenés à préciser ou uniformiser certains descripteurs de la base de données. En les résumant et en les commentant, nous en donnons quelques exemples.

\section{- Type de sol et humus}

La nomenclature du "Référentiel pédologique français" a été adoptée pour tous les relevés. Un travail de correspondance a donc été fait entre anciennes et nouvelles appellations car ces dernières sont encore peu pratiquées.

\section{- Habitats}

Types de stations et habitats sont, autant que faire se peut, mis en correspondance. Cette correspondance, utilisant en particulier le travail de Savoie (1996), est encore discutée.

\section{- Groupe d'espèces indicatrices et groupe écologique}

Chaque catalogue nomme les "groupes écologiques" (2), même similaires, de façon différente. La raison en est que les catalogues sont réalisés de façon indépendante au moins quant aux analyses statistiques mises en œuvre. Ils fournissent donc des résultats propres à chaque étude, ce qui relativise les groupes écologiques les uns par rapport aux autres mais seulement dans le cadre de ce catalogue. La Flore forestière française (Rameau et al., 1989 et 1993), elle, parle de "groupes d'espèces indicatrices" qui ont la même définition mais relèvent d'une approche un peu différente : elle utilise les nombreuses études réalisées et les synthétise, à dire d'experts, pour la France. Prenons l'exemple de la Laîche à pilules (Carex pilulifera) qu'ont trouvé quatre auteurs et, bien sûr, la Flore forestière française.

Ce Carex est ainsi classé :

\begin{tabular}{|l|c|l|}
\hline \multicolumn{1}{|c|}{ Auteur } & Code de l'auteur & \multicolumn{1}{c|}{ Signification } \\
\hline Baffalio & A & acidiphile \\
\hline Pons & acid & acidiphile \\
\hline Savoie & Am & acidiphile mésophile \\
\hline Vignes & Am & acidiphile mésophile \\
\hline Flore forestière française & Am & acidiphile de moder mésophile \\
\hline
\end{tabular}

(2) « Ensemble d'espèces végétales ayant approximativement la même amplitude par rapport à un ou plusieurs facteurs ou descripteurs écologiques" selon la définition de Delpech et al. (1985). Les facteurs écologiques sont, dans nos cas, le niveau trophique et le niveau hydrique. 
Aussi, dans la base de données, chaque groupe de fiches issues d'un catalogue est précédé d'un glossaire qui lui est propre. Par contre, les codes utilisés pour les groupes d'espèces indicatrices de la Flore forestière française sont explicités dans la notice générale : ils sont valables pour toute la France (une retranscription des codes proposés par la Flore forestière française a été rendue nécessaire).

\section{- Alerte}

Les espèces à forte valeur patrimoniale sont signalées par une coche $(\bullet)$ dans la colonne "alerte". II peut s'agir d'espèces rares ou menacées, le cas échéant protégées, d'espèces à aire de répartition restreinte ou fragmentée, d'espèces en limite d'aire quel que soit le niveau de rareté, ou d'espèces localement peu fréquentes. Ce sont des espèces pour lesquelles, en termes de conservation, la responsabilité des gestionnaires est susceptible d'être engagée. Les indications de rareté proviennent, si possible, d'une évaluation régionale ou de synthèses sur des zones plus vastes.

Ce renseignement ne s'applique qu'aux points relevés. Un extrait du tableau "alerte" donne une idée de cas possibles (figure 3).

Figure 3

EXTRAIT DU TABLEAU “ALERTE”

\begin{tabular}{|c|c|}
\hline Nom & Raisons des précautions \\
\hline Lilium pyrenaicum . . . . . . . . & assez rare, pyrénéo-ibérique et Sud-Ouest du Massif central \\
\hline Listera cordata . . . . . . . . . . & très rare (FFF) \\
\hline Lonicera alpigena . . . & rare et disséminée (FFF) \\
\hline Lonicera etrusca . . . & très localisée (méditerranéenne) \\
\hline Lonicera nigra . . & assez rare (FFF) \\
\hline Lunaria rediviva . & assez rare (FFF) \\
\hline Malus sylvestris ... & disséminée \\
\hline Narcissus pseudo-narcissus . . . & des sous-espèces ou espèces proches sont endémiques pyrénéennes \\
\hline
\end{tabular}

\section{- Les cartes de répartition}

Les coordonnées de chaque point de relevé ont été calculées à l'aide d'une table à digitaliser et d'un logiciel de géoréférencement. II en est de même d'un certain nombre de points géographiques remarquables pour permettre à l'utilisateur de caler le calque fourni. Au point de relevé est associé son numéro, au point géographique est associé son nom tel qu'il figure sur la carte de l'Institut géographique national.

\section{LA RÉALISATION}

Le passage des fichiers des relevés vers un format informatique différent, les compléments (nom des types de stations, référence des cartes IGN, statut d'alerte...), le géoréférencement des points, la mise au point du programme de rapprochement des fichiers puis le tri et la mise en forme des documents ont constitué des phases longues. II a fallu prévoir les corrections 
dans les divers fichiers de base, les validations et harmonisations de tous les noms issus de nomenclatures variées... Le contenu des fichiers est montré ci-dessous et p. 54 .

Une application informatique originale a été mise au point. Pour créer la base de données, trois fichiers principaux (tous sous Windows) sont associés :

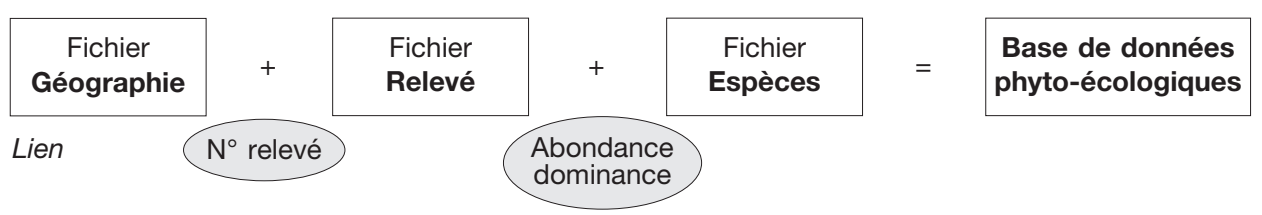

\section{EXTRAIT DU FICHIER "RELEVÉ”}

\begin{tabular}{|c|c|}
\hline 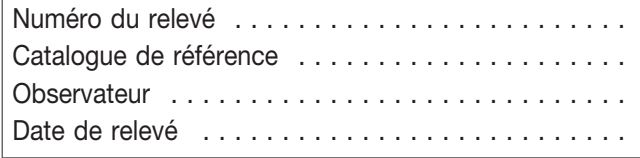 & $\begin{array}{l}65 \\
\text { Pré-Pyrénées du Comminges } \\
\text { D. Pons } \\
26 / 06 / 1993\end{array}$ \\
\hline 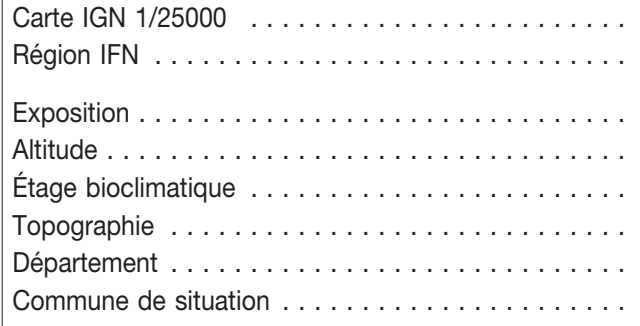 & $\begin{array}{l}19.46 \text { O } \\
\text { Pré et Petites-Pyrénées } \\
\mathrm{N} \\
410 \\
\text { collinéen moyen } \\
\text { mi-pente } \\
31 \\
\text { Pointis-Inard }\end{array}$ \\
\hline $\begin{array}{l}\text { Forêt/Propriété } \\
\text { Canton/Parcelle }\end{array}$ & \\
\hline 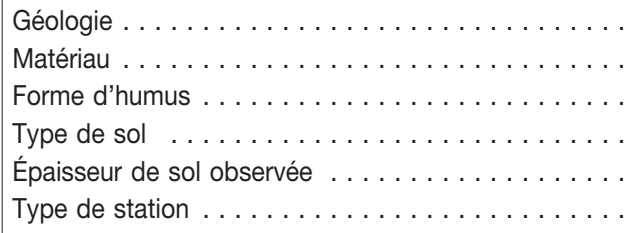 & $\begin{array}{l}\text { marne ou calcaire marneux } \\
\text { altérite décarbonatée, sans éléments grossiers carbonatés } \\
\text { mésomull } \\
\text { brunisol ou néoluvisol mésosaturé } \\
\text { plus de } 120 \mathrm{~cm} \\
3\end{array}$ \\
\hline $\begin{array}{l}\text { Habitat } \\
\text { Code CORINE de l'habitat } \ldots \ldots \ldots \ldots \ldots \ldots\end{array}$ & 41.22 \\
\hline $\begin{array}{l}\text { Abies alba } A \\
\text { Abies alba ab } \\
\text { Abies alba } h \\
\text { Acer campestre } A \\
\text { Acer campestre } a b \\
\text { Acer campestre } h \ldots \ldots \ldots \ldots \ldots \ldots \ldots \ldots \ldots \ldots \\
\text { Acer pseudoplatanus } h\end{array}$ & 1 \\
\hline
\end{tabular}


M. BARTOLI - G. LARGIER - S. ABGRALL - E. LUXCEY

EXTRAIT DU FICHIER “ESPÈCES”

\begin{tabular}{|c|c|c|c|c|c|c|c|c|c|c|}
\hline Nom correct & Code Type & Larrieu & Baffalio & Pons & Vignes & $\begin{array}{l}\text { Savoie } \\
\text { Coll }\end{array}$ & $\begin{array}{l}\text { Savoie } \\
\text { Mont }\end{array}$ & $\begin{array}{l}\text { FFF } \\
\text { Mont }\end{array}$ & $\begin{array}{l}\text { FFF } \\
\text { Coll }\end{array}$ & Alerte \\
\hline Abies alba $\ldots \ldots$ & aalw $A$ & & & $\begin{array}{c}\text { non } \\
\text { classée }\end{array}$ & & tla Ihs & tla lhs & tla & tla & \\
\hline 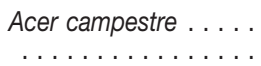 & acaw $\mathrm{A}$ & G7m & Ncmx & $\begin{array}{l}\text { non } \\
\text { classée }\end{array}$ & $\mathrm{ncm}$ & $\mathrm{ccl} \mathrm{m}$ & $\mathrm{ccl} \mathrm{m}$ & b & b & \\
\hline Acer monspessulanum & acmo A & & & & & $\mathrm{cmx}$ & $\mathrm{cmx}$ & & $\mathrm{x} / \mathrm{xx} \mathrm{BB}$ & \\
\hline Acer opalus $\ldots . .$. & aopw A & & Ncmx & & & $c X$ & $c X$ & $\mathrm{x} / \mathrm{xx} \mathrm{BB}$ & $\mathrm{x} / \mathrm{xx} \mathrm{BB}$ & - \\
\hline $\begin{array}{c}\text { Acer platanoides } \ldots \\
\ldots \ldots \ldots \ldots\end{array}$ & aplw A & G11 & & & $\begin{array}{l}\text { non } \\
\text { classée }\end{array}$ & $\mathrm{n}$ Ihs & $\mathrm{n}$ Ihs & $\mathrm{n}$ am & $\mathrm{n}$ am & - \\
\hline $\begin{array}{c}\text { Acer pseudoplatanus } \\
\ldots \ldots \ldots \ldots \ldots\end{array}$ & apsw A & $\begin{array}{l}\text { non } \\
\text { classée }\end{array}$ & & $\begin{array}{l}\text { non } \\
\text { classée }\end{array}$ & & nma hs & nma hs & $\mathrm{n} \mathrm{la}$ & $\mathrm{n} \mathrm{la}$ & \\
\hline Achillea millefolium & acmi $\mathrm{h}$ & & & & & nla $m x$ & nla $m x$ & $\mathrm{nN} \mathrm{m}$ & $\mathrm{nN}$ & \\
\hline Acinos arvensis .. & caac $\mathrm{h}$ & & & & & $c X$ & $c X$ & & & \\
\hline Aconitum napellus & acna $\mathrm{h}$ & & & & & $\mathrm{nh}$ & $\mathrm{nh}$ & & & - \\
\hline Aconitum vulparia & acvu $\mathrm{h}$ & & & & & $\mathrm{n}$ hs & $\mathrm{n}$ hs & H sc & H sc & - \\
\hline Actaea spicata $\ldots$ & acsp $\mathrm{h}$ & & & & & nma lhs & nma lhs & $\mathrm{H} \mathrm{sc}$ & $\mathrm{H}$ sc & - \\
\hline Adenostyles alliariae & adal $\mathrm{h}$ & & & & & a hs & a hs & & & \\
\hline Agrostis capillaris & agca $h$ & G10 & & acid & $\mathrm{Am}$ & $\mathrm{mA} \mathrm{m}$ & $\mathrm{mA} \mathrm{m}$ & $\mathrm{Am}$ & $\mathrm{Am}$ & \\
\hline
\end{tabular}

\section{EXTRAIT DU FICHIER “GÉOGRAPHIE”}

\section{Station}

\begin{tabular}{|l|c|r|}
\hline \multicolumn{2}{|l|}{$\mathrm{N}^{\circ}$ du point } & 883 \\
\hline \multicolumn{2}{|l|}{ Carte IGN } & 18470 \\
\hline $\begin{array}{l}\text { Coordonnées } \\
\text { Lambert III (m) }\end{array}$ & $\mathrm{X}$ & 444074 \\
\cline { 2 - 3 } & $\mathrm{Y}$ & 3069169 \\
\hline GT ONF & 123260 \\
\hline
\end{tabular}

\begin{tabular}{|l|c|c|}
\hline \multicolumn{2}{|l|}{ Repère } & \multicolumn{2}{|l|}{265} \\
\hline$N^{\circ}$ du point & 18470 \\
\hline Carte IGN & Sommet \\
\hline Type & & Cap de Cros \\
\hline Nom & X & 444263 \\
\hline $\begin{array}{l}\text { Coordonnées } \\
\text { Lambert III (m) }\end{array}$ & Y & 3069892 \\
\hline
\end{tabular}

\section{L'UTILISATION ET LA GESTION DE LA BASE DE DONNÉES PHYTO-ÉCOLOGIQUES}

Informatisée et géoréférencée, la base de données permet tris et sélections. Demander les points situés dans les forêts domaniales de tel ou tel département sur lesquels on trouve une ou des espèces données, celles avec une certaine valeur patrimoniale..., extraire la position cartographique et la commune de situation de tous les luvisols... est possible. Ainsi rassemblés les fichiers actuels permettent de mieux analyser des études d'autécologie, de fournir des cartes de répartition avec toutes les indications écologiques utiles.

Une telle base cartographique de données a encore les imperfections d'un prototype. Pour l'instant, le bureau d'études de l'ONF à Toulouse la gère. À terme, le Conservatoire botanique pyrénéen en assurera le suivi (entrée de nouvelles études, actualisation nomenclaturale...) en l'intégrant dans une base de données beaucoup plus vaste et en la mettant en relation avec d'autres bases comme celle de Brisse et Grandjouan (1971). 


\section{CONCLUSIONS}

Utilisant des données d'écologie pyrénéenne déjà disponibles, cette base de données est un repère assez précis pour tous les gestionnaires de terrain et permet une approche de la répartition d'espèces, de sols... La phase d'investissement méthodologique et de mise au point des outils informatiques est terminée.

De nombreux utilisateurs peuvent être intéressés par cette reprise des données initiales d'un catalogue des types de stations forestières. Cet usage conforte la méthode permettant de réaliser des catalogues dans lesquels une forte place est accordée aux écosystèmes et à leur valeur patrimoniale au travers, le plus souvent, des espèces végétales.

Pour les praticiens forestiers, la fiche de la base de données est la page d'un livre dont les illustrations sont sur le terrain. Même si cette page n'est qu'un point, celui décrit par l'auteur du catalogue, la connaissance de ses caractéristiques phyto-écologiques devrait singulièrement faciliter et rendre plus sûr le nécessaire travail ultérieur de cartographie.
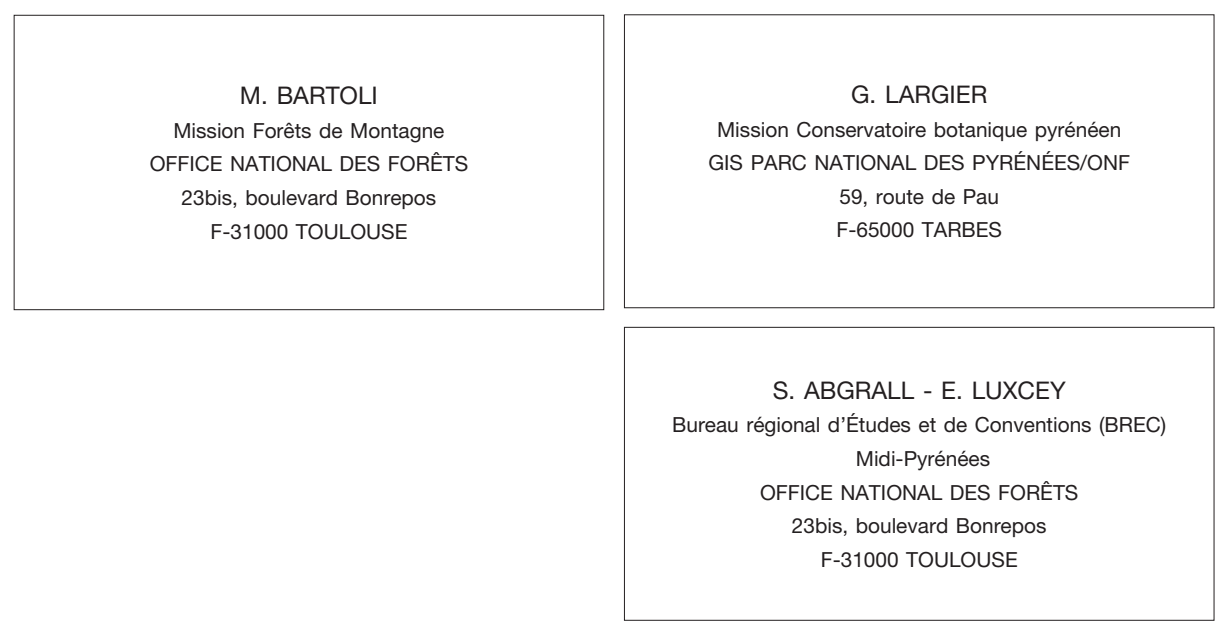

Le travail présenté a été financé par le Département "Études et expertises" de l'Office national des Forêts.

\section{BIBLIOGRAPHIE}

BAFFALIO (S.). - Approche stationnelle d'une hêtraie sur roches calcaires : le massif des Arbailles (Pyrénées occidentales). - ONF, 1995. - 43 p. (Mémoire cert. spéc. typ. sta. for.).

BRÊTHES (A.). - La Typologie des stations forestières. Recommandations méthodologiques. - Revue forestière française, vol. XLI, $\mathrm{n}^{\circ}$ 1, 1989, pp. 7-27.

BRISSE (H.), GRANDJOUAN (G.). - Formulaire floristique des végétaux vasculaires de la France. Notice d'emploi. - CNRS CEPE, 1971. - 20 p.

DELPECH (R.), DUMÉ (G.), GALMICHE (P.). - Typologie des stations forestières. Vocabulaire. - Paris : IDF, 1985. $-243 \mathrm{p}$. 
DUPOUEY (J.-L.), BECKER (M.), BERT (D.), CADEL (G.), LEFÈVRE (Y.), PICARD (J.-F.), THIMONIER (A.). - Évolution récente des sols, de la végétation et de la productivité des forêts de montagne françaises. Végétation et sols de montagne : diversité, fonctionnement et évolution. Colloque de Grenoble, 8-13 juillet 1996. - Écologie, vol. 29, n 1-2, 1998, pp. 341-350.

GOYHENEIX (S.). - Typologie des stations de la forêt communale d'Oloron-Sainte-Marie. - Mémoire ENITEF, 1995. - $56 \mathrm{p}$.

LARGIER (G.). - Approche stationnelle d'une hêtraie montagnarde, la forêt domaniale de Cagire, Haute-Garonne. - ONF, 1988. - 48 p. et annexes (Mémoire cert. spéc. typ. sta. for.).

LARRIEU (L.). - Étude des types de stations des coteaux béarnais sous-pyrénéens d'Oloron à Nay. - CRPF Aquitaine, 1994. - 48 p. (Mémoire cert. spéc. typ. sta. for.).

PONS (D.). - Les Stations forestières du massif de Pointis-Inard. - ONF, 1993. - 45 p. (Mémoire cert. spéc. typ. sta. for.).

RAMEAU (J.-C.), MANSION (D.), DUMÉ (G.). - Flore forestière française. Tome 1 : Plaines et collines. - Paris : IDF, 1989. - 1785 p.

RAMEAU (J.-C.), MANSION (D.), DUMÉ (G.). - Flore forestière française. Tome 2 : Montagnes. - Paris : IDF, 1993. - $2421 \mathrm{p}$.

SAVOIE (J.-M.). - Les Types de stations forestières des Pyrénées centrales. Front pyrénéen et Haute chaîne. Vallée d'Aure, Haut Comminges et Couserans. - ONF Midi-Pyrénées, 1995. - 507 p.

SAVOIE (J.-M.). - Rapprochement entre types de stations et habitats forestiers des Pyrénées centrales. - ONF, 1996. - $107 \mathrm{p}$.

TIERLE (G.). - La Régénération du Sapin dans les Hautes-Pyrénées. - Mémoire ENITEF, 1978. - 68 p. et annexes.

VIGNES (B.). - Approche stationnelle des forêts communales ariégeoises des Petites Pyrénées. - ONF Ariège, 1993. - 79 p. (Mémoire cert. spéc. typ. sta. for.).

\section{UNE BASE CARTOGRAPHIQUE DE DONNÉES PHYTO-ÉCOLOGIQUES ISSUE DES RELEVÉS DE CATALOGUES DE TYPES DE STATIONS FORESTIÈRES (Résumé)}

Un catalogue des types de stations forestières est la synthèse de centaines de relevés phyto-écologiques. Associées au point de relevé géoréférencé à l'aide d'un logiciel de cartographie (SIG) et complétées (stations, habitats...), les données initiales permettent de créer une base de données originale. Elle a pour objectifs d'être un point de repère pour les forestiers de terrain et une nouvelle source de données pour les scientifiques. Elle permet d'améliorer les connaissances de la répartition des espèces végétales, des sols ou des habitats.

Servant de prototype et utilisant les données de 5 catalogues couvrant 160000 ha de forêts des Pyrénées centro-occidentales, une première base de données phyto-écologiques gère environ 170000 données issues de 1408 relevés, soit environ un point tous les 120 ha de forêt. À la demande d'un utilisateur, elle permet de fournir cartes et fichiers complets associés aux points de relevés répondant à la requête.

\section{A CARTOGRAPHIC DATA BASE DERIVED FROM PHYTOECOLOGICAL DATA RECORDS IN SITE TYPE CATA- LOGUES (Abstract)}

A site type catalogue is a synopsis of hundreds of pedological and botanical data records. An original database has been set up by attaching geographic references to the initial data using the GIS plus additional information (site type, habitat etc.). Its purpose is to serve as a reference point for foresters and an additional source of data for scientists. It is a means for enhancing knowledge on the chorology of taxons, soils and habitats.

As a prototype, a first phyto-ecological data base has been built from five catalogues relating to the Pyrenees (160 000 ha of forests, 170000 data items derived from 1408 records, i.e., approximately one point recorded every 120 ha). At the user's request, the maps and complete records for the geographical coordinates associated with the request can be provided. 